\title{
Inherited PAX6, NF1 and OTX2 mutations in a child with microphthalmia and aniridia
}

\author{
R Alex Henderson*, ${ }^{\star}$, Kathy Williamson ${ }^{2}$, Sally Cumming ${ }^{3}$, Michael P Clarke ${ }^{1}$, \\ Sally Ann Lynch ${ }^{1}$, Isabel M Hanson ${ }^{4}$, David R FitzPatrick ${ }^{2}$, Sanjay Sisodiya ${ }^{5}$ and \\ Veronica van Heyningen ${ }^{2}$
}

\begin{abstract}
${ }^{1}$ Newcastle upon Tyne Hospitals NHS Trust, Newcastle upon Tyne, NE1 4LP, UK; ${ }^{2}$ MRC Human Genetics Unit, Western General Hospital, Edinburgh, EH4 2XU, UK; ${ }^{3}$ Regional Molecular Genetics Laboratory, Addenbrooke's Hospital, Cambridge CB2 2QQ UK; ${ }^{4}$ University of Edinburgh School of Molecular and Clinical Medicine, Western General Hospital, Edinburgh, EH4 2XU, UK; ${ }^{5}$ Department of Clinical and Experimental Epilepsy, Institute of Neurology, UCL, Queen Square, London WC1N 3BG, UK
\end{abstract}

A girl with aniridia, microphthalmia, microcephaly and café au lait macules was found to have mutations in PAX6, NF1 and OTX2. A novel PAX6 missense mutation (p.R38W) was inherited from her mother whose iris phenotype had not been evident because of ocular neurofibromatosis. Analysis of the NF1 gene in the proband, prompted by the mother's diagnosis and the presence of café au lait spots, revealed a nonsense mutation (p.R192X). Subsequently an OTX2 nonsense mutation (p.Y179X) was identified and shown to be inherited from her father who was initially diagnosed with Leber's congenital amaurosis. Since individual mutations in PAX6, OTX2 or NF1 can cause a variety of severe developmental defects, the proband's phenotype is surprisingly mild. This case shows that patients with complex phenotypes should not be eliminated from subsequent mutation analysis after one or even two mutations are found.

European Journal of Human Genetics (2007) 15, 898-901; doi:10.1038/sj.ejhg.5201826; published online 4 April 2007

Keywords: aniridia; microphthalmia; gene interactions

\section{Introduction}

Abnormalities in ocular development can lead to a variety of structural congenital eye defects including anterior segment anomalies and the microphthalmia, anophthalmia and coloboma spectrum. ${ }^{1-3}$ These conditions often show reduced penetrance and phenotypic variability and join the growing number of human genetic diseases that cannot easily be classified as single-gene disorders. ${ }^{4,5}$ There is increasing recognition that variable penetrance and phenotype are associated with mutations in multiple

${ }^{*}$ Correspondence: Dr RA Henderson, Northern Genetics Service, Institute of Human Genetics, Centre for Life, Newcastle upon Tyne NE1 4BZ, UK. Tel: + 44191241 8750; Fax: + 44191241 8799;

E-mail: alexhenderson@doctors.org.uk

Received 27 November 2006; revised 16 February 2007; accepted 21 February 2007; published online 4 April 2007 interacting genes working within common developmental pathways. ${ }^{5-7}$ Full clinical assessment and thorough molecular investigation of affected individuals and families are essential for a proper understanding of the underlying developmental biology and genetic interactions.

Here we describe a girl with dominant mutations in three genes that affect eye development, NF1, OTX2 and PAX6. NF1 encodes a multifunctional cytoplasmic signalling protein and is expressed ubiquitously during development, including in early eye tissue. ${ }^{8}$ Neurofibromatosis type I (NFI) patients can have a variety of eye anomalies including Lisch nodules, optic gliomas and anterior segment defects. ${ }^{9}$ PAX6 and OTX2 encode DNA binding proteins that regulate transcription and have highly specific neural and ocular expression patterns. ${ }^{3}$ OTX2 has a homeodomain; PAX6 has a homeodomain and a paired domain. ${ }^{3}$ PAX6 mutations typically cause aniridia, but 
some are associated with microphthalmia. ${ }^{10}$ OTX2 mutations cause a range of eye defects varying from relatively mild to bilateral anophthalmia. ${ }^{11}$

\section{Materials and methods Case reports}

The proband (Figure 1) was born at gestational week 38 with a birth weight of $2730 \mathrm{~g}$ (90th centile). She was microcephalic (OFC $<3$ rd centile) and had a microphthalmic right eye and a fixed dilated left pupil. Ophthalmological examination soon after birth revealed right microcornea (3-mm diameter which compares to a normal cornea diameter at birth of approximately $10 \mathrm{~mm}$ ) with loss of the central portion of the iris, prominent ciliary processes, inferior dislocation of the lens and a cataract. The left eye had a normal sized cornea (10.5-mm diameter), typical aniridia with an iris remnant and a clear lens. The left fundus showed a vertically oval optic disc, foveal hypoplasia, retinal white spots near the macula and a possible temporal epiretinal membrane. Nystagmus was present. Later assessment indicated left hypermetropia with acuity of $6 / 48$ and no vision in the right eye. In the first year, multiple café au lait macules and axillary freckling developed which, together with a family history, provided a clinical diagnosis of NFI.

At age 5 her global developmental age was about 4 years. She attends a mainstream school with a unit for children with special needs. There were no behavioural difficulties and no specific language or motor abnormalities.

On MRI brain scan, which was performed at the age of 3, the pineal gland was not visible and the foramina of Magendie were enlarged. Absence of the pineal gland is associated with PAX6 mutation. ${ }^{12}$

The proband's mother had NFI (Figure 1) with typical eye defects comprising retinal fibroma, optic nerve glioma and gross Lisch nodules on the iris. Both eyes were of normal size but had small corneas (right 9.5-mm diameter, left 10-mm diameter; these compare to a normal adult cornea diameter of approximately $12 \mathrm{~mm}$ ), cataracts, optic nerve hypoplasia and nystagmus. There was mild iris stromal hypoplasia with normal-sized pupils. Visual acuity at 10 years of age was $6 / 24$ for each eye although this has deteriorated due to cataracts and optic glioma.

The proband's father (patient $4 \mathrm{~b}$ in Ragge et $a^{11}$ ) was diagnosed with Leber's congenital amaurosis (LCA). He had typical LCA retinal features of pale optic discs, thin vessels, atrophic maculae, mid-peripheral pigment clumps and nystagmus. The proband's father also had features atypical
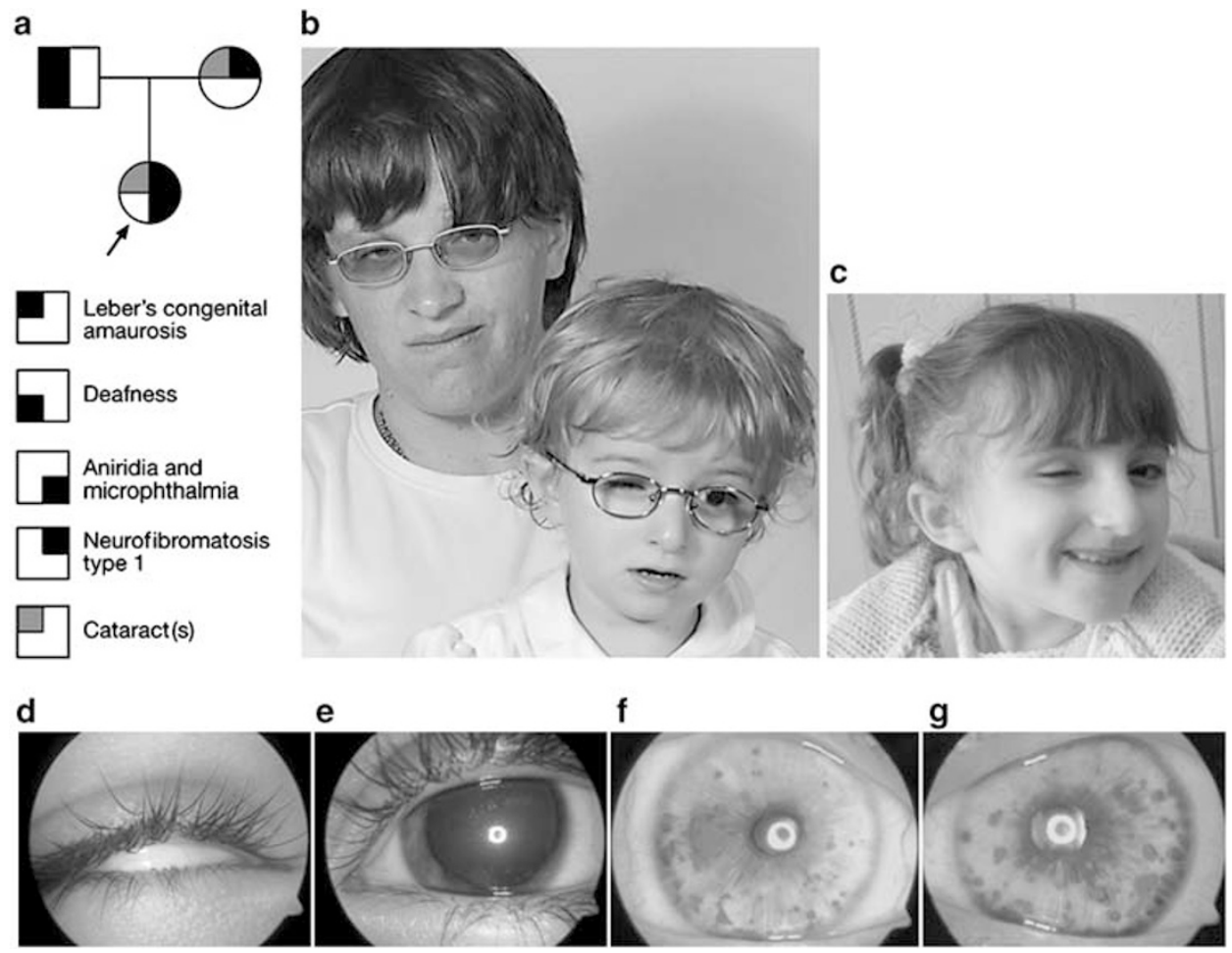

Figure 1 Phenotypes of the proband (indicated with arrow) and her mother. (a) The pedigree, showing the proband inheriting neurofibromatosis and cataracts from her mother. (b) The mother, with small neurofibroma-like skin lesions, and the proband, aged 2.5 years, with right microphthalmia. (c) The proband aged 5 years 3 months. (d) Microphthalmic right eye and (e) aniridia in the left eye of the proband. (f) Right and (g) left eye of the mother, with Lisch nodules overlying visible iris tissue. 
of LCA including bilateral mild microphthalmia, mild microcornea (11-mm diameters) and iridocorneal synechiae. Intraocular pressure was normal and visual acuity was $6 / 60$. He was deaf in the right ear probably secondary to childhood meningitis.

\section{Molecular analysis}

Genomic DNA was extracted as described in Ragge et al ${ }^{11}$ and screened for point mutations in the entire coding sequences of PAX6, NF1 and OTX2. Pre-screening of PAX6 by DHPLC ${ }^{4}$ (Transgenomic Ltd, Cramlington, UK) revealed heterozygosity for exon 5 which was analysed by direct sequencing and restriction enzyme digestion. ${ }^{13}$ OTX2 and NF1 were screened by direct sequencing. ${ }^{11,14}$ Sequences were bidirectional and generated from duplicate PCRs. Mutation numbering is in accordance with The Human PAX6 Allelic Variant Database reference sequence (http:// pax6.hgu.mrc.ac.uk/) (PAX6), GenBank AY796305 (NF1) and GenBank NM_172337 (OTX2).

\section{Results}

On the basis of the proband's eye phenotype, her PAX6 gene was screened and found to contain a heterozygous exon 5 mutation c.474C $>$ T (p.R38W missense mutation; Figure $2 \mathrm{a}$ ). Since the proband has café au lait spots and her mother has NFI, the proband's NF1 gene was sequenced, revealing a heterozygous mutation in exon $4 \mathrm{~b}(\mathrm{c} .574 \mathrm{C}>\mathrm{T}$; p.R192X nonsense mutation; Figure 2b). The proband's DNA was also included in a large-scale screen for OTX2 mutations which uncovered a heterozygous exon 3 change c.708T $>$ A (p.Y179X nonsense mutation, Figure 2c). The OTX2 mutation was not observed in 96 ethnically matched controls. The frequencies of the PAX6 and NF1 mutations in the general population are unknown.

From analysis of genotype and phenotype, the NF1 and PAX6 mutations were maternally inherited (Figure 2d) and the OTX2 mutation was paternally inherited. The father, his sister and his mother all have the OTX2 mutation (family 4 in Ragge et $a l^{11}$ ). The father's sister is blind, has severe bilateral microphthalmia, profound developmental delay and seizures. The father's mother, who has retinopathy with pigment clumps, is a gonosomal mosaic, with a low level of the OTX2 mutant allele in her blood DNA. ${ }^{11}$

\section{Discussion}

The PAX6 R38 residue is invariant in all known paired domains and directly contacts DNA. ${ }^{13}$ The highly nonconservative substitution of arginine by tryptophan would be predicted to impair PAX6 function. This mutation was also observed in a man with ocular coloboma (unpublished data). The two nonsense mutations may have different consequences. Based on the current model that nonsensemediated decay (NMD) degrades mammalian mRNAs
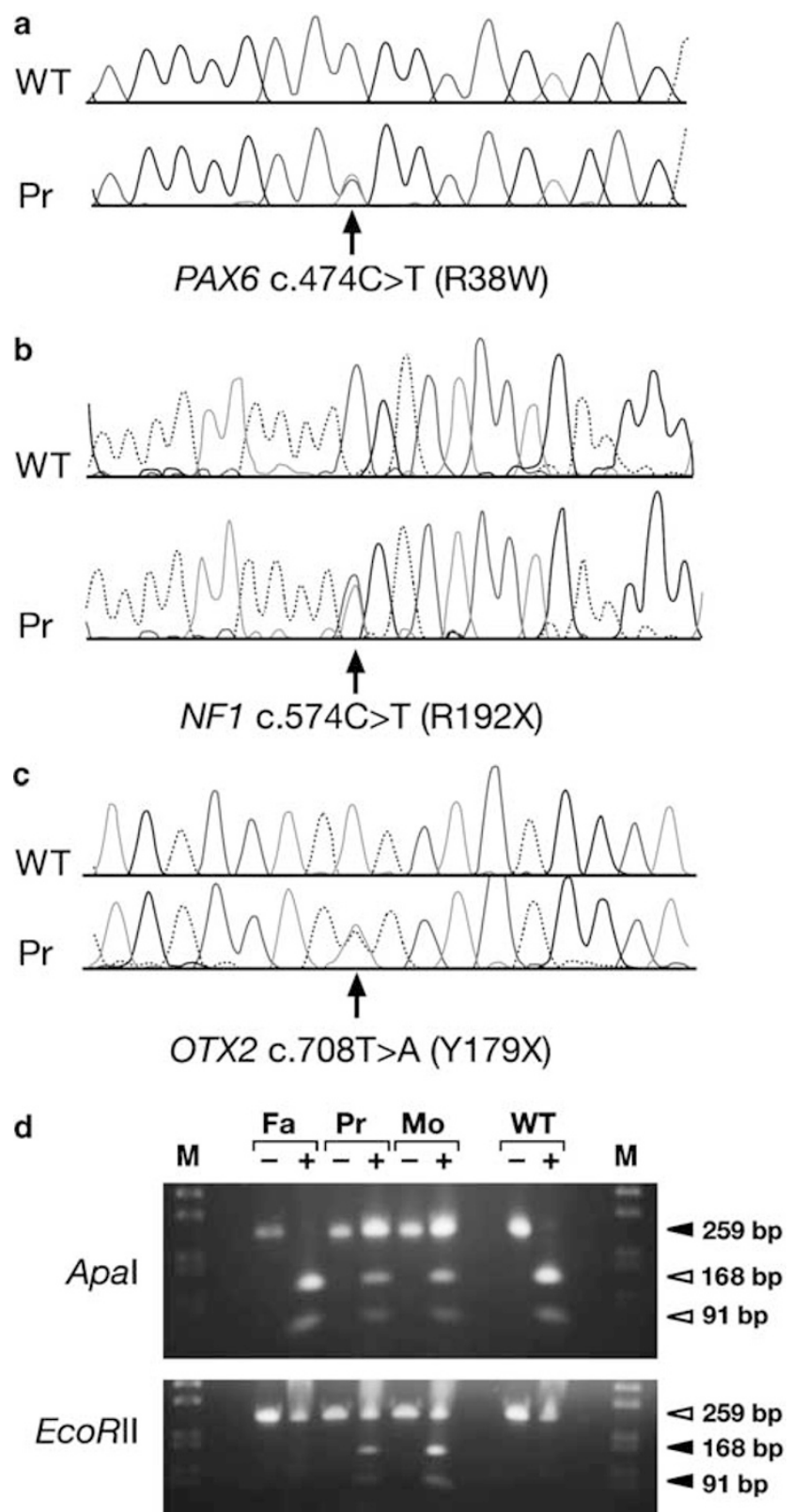

Figure 2 Heterozygous mutations in PAX6, NF1 and OTX2. (a) PAX6 exon $5 \mathrm{C}>\mathrm{T}$ transition (arrow) in the proband. (b) NF1 exon $4 \mathrm{~b}$ $\mathrm{C}>\mathrm{T}$ transition (arrow) in the proband. (c) OTX2 T $>$ A transversion (arrow) in the proband. (d) PAX6 exon 5 PCR products undigested (-) and digested $(+)$ with Apal (top) or EcoRII (bottom). Digestion products are indicated as normal (open arrowheads) or mutant (solid arrowheads). The proband and mother have both normal and mutant fragments, whereas the father has only normal fragments. WT, wild type control; Pr, proband; Fa, father; Mo, mother; M, molecular size marker.

containing premature stop codons located more than about 50 bases upstream of the last exon-exon junction, NMD is predicted for the NF1 mutation, creating a functional null, but not the OTX2 mutation, which is predicted to generate a prematurely truncated protein. $3,11,15$ 
The proband's mild phenotype is surprising considering the severity of developmental abnormalities associated with single mutations in PAX6, NF1 and OTX2. The forebrain patterning gene Hes $x 1$ is activated by Otx 2 and repressed by Pax6, ${ }^{16}$ and the reciprocal expression of Pax6 and $0 t \times 2$ in early neural tissue may also indicate mutual regulation. ${ }^{17}$ Reduced levels of both proteins may result in amelioration of the associated anomalies.

This family illustrates the importance of rigorous clinical assessment and extensive mutation screening in complex phenotypes.

\section{Acknowledgements}

We thank the family for their involvement in this study.

\section{References}

1 FitzPatrick DR, Heyningen V: Developmental eye disorders. Curr Opin Genet Dev 2005; 15: 348-353.

2 Graw J: The genetic and molecular basis of congenital eye defects. Nat Rev Genet 2003; 4: 876-888.

3 Hever A, Williamson $\mathrm{K}$, van Heyningen V: Developmental malformations of the eye: the role of PAX6, SOX2 and OTX2. Clin Genet 2006; 69: 459-470.

4 Morrison D, FitzPatrick D, Hanson I et al: National study of microphthalmia, anophthalmia, and coloboma (MAC) in Scotland: investigation of genetic aetiology. J Med Genet 2002; 39: $16-22$.

5 Ming J, Muenke M: Multiple hits during early embryological development: digenic diseases and holoprosencephaly. Am J Human Genet 2002; 71: 1017-1032.
6 Carrasquillo MM, McCallion AS, Puffenberger EG, Kashuk CS Nouri N, Chakravarti A: Genome-wide association study and mouse model identify interaction between RET and EDNRB pathways in Hirschsprung disease. Nat Genet 2002; 32: 237-244.

7 Badano JL, Leitch CC, Ansley SJ et al: Dissection of epistasis in oligogenic Bardet-Biedl syndrome. Nature 2006; 439: 326-330.

8 Daston MM, Ratner N: Neurofibromin, a predominantly neuronal GTPase activating protein in the adult, is ubiquitously expressed during development. Dev Dyn 1992; 195: 216-226.

9 Beauchamp GR: Neurofibromatosis type 1 in children. Trans Am Ophthalmol Soc 1995; 93: 445-472.

10 Tzoulaki I, White IM, Hanson IM: PAX6 mutations: genotypephenotype correlations. BMC Genet 2005; 6: 27.

11 Ragge N, Brown A, Poloschek C et al: Heterozygous mutations of the OTX2 gene cause a syndrome of ocular malformations. Am J Human Genet 2005; 76: 1008-1022.

12 Mitchell TN, Free SL, Williamson KA et al: Polymicrogyria and absence of pineal gland due to PAX6 mutation. Ann Neurol 2003; 53: $658-663$.

13 Hanson I, Churchill A, Love J et al: Missense mutations in the most ancient residues of the PAX6 paired domain underlie a spectrum of human congenital eye malformations. Hum Mol Genet 1999; 8: 165-172.

14 Toliat MR, Erdogan F, Gewies A et al: Analysis of the NF1 gene by temperature gradient gel electrophoresis reveals a high incidence of mutations in exon 4b. Electrophoresis 2000; 21: 541-544.

15 Lejeune F, Maquat L: Mechanistic links between nonsensemediated mRNA decay and pre-mRNA splicing in mammalian cells. Curr Opin Cell Biol 2005; 17: 309-315.

16 Spieler D, Baumer N, Stebler J et al: Involvement of Pax6 and Otx2 in the forebrain-specific regulation of the vertebrate homeobox gene ANF/Hesx1. Dev Biol 2004; 269: 567-579.

17 Zuber ME, Gestri G, Viczian AS, Barsacchi G, Harris WA: Specification of the vertebrate eye by a network of eye field transcription factors. Development 2003; 130: 5155-5167. 